\title{
EXISTENCE OF POSITIVE ENTIRE SOLUTIONS OF SEMILINEAR ELLIPTIC EQUATIONS ON $\mathbf{R}^{\mathbf{N}}$
}

\author{
N. HIRANO
}

\section{INTRODUCTION}

In the present paper we are concerned with positive solutions of the following problem:

$$
\left\{\begin{array}{l}
-\Delta u+u=g(x, u), \quad x \in R^{N}, \\
u \in H^{1}\left(R^{N}\right), \quad N \geq 3
\end{array}\right.
$$

where $g: R^{N} \times R \rightarrow R$ is a continuous mapping. Recently, the existence of positive solutions of the semilinear elliptic problem

$$
\left\{\begin{array}{l}
-\Delta u+u=Q(x)|u|^{p-1} u, \quad x \in R^{N}, \\
u \in H^{1}\left(R^{N}\right), \quad N \geq 2,
\end{array}\right.
$$

has been studied by several authors, where $1<p$ for $N=2,1<p<$ $(N+2) /(N-2)$ for $N \geq 3$ and $Q(x)$ is a positive bounded continuous function. If $Q(x)$ is a radial function, we can find infinity many solutions of problem $\left(P_{Q}\right)$ by restricting our attention to the radial functions (cf. $\left.[2,5]\right)$. If $Q(x)$ is nonradial, we encounter a difficulty caused by the lack of a compact embedding of Sobolev type. To overcome this kind of difficulty, P. L. Lions developed the concentrate compactness method [8,9], and established the following result: Assume that $\lim _{|x| \rightarrow \infty} Q(x)=\bar{Q}(>0)$ and $Q(x) \geq \bar{Q}$ on $R^{N}$. Then the problem $\left(P_{Q}\right)$ has a positive solution. This result is based on the observation that the ground state level $c_{Q}$ of the functional

$$
I_{Q}(u)=\frac{1}{2} \int_{R^{N}}\left(|\nabla u|^{2}+|u|^{2}\right) d x-\frac{1}{p+1} \int_{R^{N}} Q(x)|u|^{p+1} d x
$$

1991 Mathematics Subject Classification. Primary: 35J60, 35J65.

Key words and phrases. Positive solutions, concentrate compactness method, homology groups.

Received: January 10, 1996.

(C) 1996 Mancorp Publishing, Inc. 
is lower than that of

$$
I^{\infty}(u)=\frac{1}{2} \int_{R^{N}}\left(|\nabla u|^{2}+|u|^{2}\right) d x-\frac{1}{p+1} \int_{R^{N}}|u|^{p+1} d x,
$$

then, under additional conditions on $g$, there exists a positive solution of (P) (cf. Ding and Ni [5], Stuart [14]). In [3], Cao proved the existence of a positive solution of $\left(P_{Q}\right)$ for the case $c_{Q} \leq c_{\bar{Q}}$ under the hypothesis that $\lim _{|x| \rightarrow \infty} Q(x)=\bar{Q}$ and $Q(x) \geq 2^{(1-p) / 2} \bar{Q}$ on $R^{N}$. The difficultly in treating the case $c_{Q}=c_{\bar{Q}}$ is caused by the fact that we can not apply the concentrate compactness method directly. The argument in [3] is based on Lagrange's method of indeterminate coefficients. That is, if we find a solution $u$ of the minimizing problem

$$
\begin{aligned}
\inf \left\{\left\{I_{Q}(u): u \in V_{\lambda}\right\},\right. & \\
V_{\lambda} & =\left\{\left\{u \in H^{1}\left(R^{N}\right), u>0, \int_{R^{N}} Q(x)|u|^{p+1} d x=1\right\}\right\},
\end{aligned}
$$

then $c u$ is a solution of $\left(P_{Q}\right)$ for some $c>0$. Lagrange's method does not work if $g$ is not the form $Q(x) t^{p}$. Our purpose in this paper is to consider the existence of a positive solution of $(P)$ for $g$ satisfying $\lim _{|x| \rightarrow \infty} g(x, t)=$ $|t|^{p-1} t$. Our method employed here is based on the singular homology theory.

Throughout this paper, we assume that $g \in C^{1}(R) \cap C^{2}(R \backslash\{0\})$ and we impose the following conditions on $g$ :

(g1) There exists a positive number $d<1$ such that

$$
\begin{aligned}
& -d t+(1-d)|t|^{p-1} t \leq g(x, t) \leq d t+(1+d)|t|^{p-1} t \\
& \quad \text { for all }(x, t) \in R^{N} \times[0, \infty) ;
\end{aligned}
$$

(g2) there exists a positive number $C$ such that

$$
\begin{aligned}
& \left|g_{t}(x, 0)\right|<1 \text { and } 0<t^{3} g_{t t}(x, t)<C\left(1+|t|^{p+1}\right) \\
& \text { for all }(x, t) \in R^{N} \times(0, \infty) ; \\
& \qquad \lim _{|x| \rightarrow \infty} g(x, t)=|t|^{p-1} t
\end{aligned}
$$

uniformly on bounded intervals in $[0, \infty)$,

where $1<p<(N+2) /(N-2)$ and $g_{t}(\cdot, \cdot)$ stands for the derivative of $g$ with respect to the second variable. 
Remark 1. (1) Throughout the rest of this paper, we assume for the simplicity of the proofs that $g(x,-t)=-g(x, t)$ for $(x, t) \in R^{N} \times[0, \infty)$. Since we are concerned with positive solutions, this assumption does not effect our result. By this assumption, the functional $I$ is even and if $u$ is a critical point of $I,-u$ is also a critical point of $I$. (2) Functions of the form $g(x, t)=\sum_{i=1}^{m} q_{i}(x) t^{i}+q_{p}(x) t^{p}$ satisfy (g1) and (g2) if $m$ is a positive integer with $m<p, q_{i}(x)(1 \leq i \leq m)$ are sufficiently small and $\left|q_{p}(x)-1\right|<1+d$. (g3) is satisfied if $\lim _{|x| \rightarrow \infty} q_{i}(x)=0$ for $1 \leq i \leq p-1$ and $\lim _{|x| \rightarrow \infty} q_{p}(x)=1$.

Theorem. Suppose that $(g 2)$ and $(g 3)$ hold. Then there exists $d_{0}>0$ such that if $(g 1)$ holds with $d<d_{0}$, then the problem $(P)$ has a positive solution.

\section{Preliminaries}

Throughout the rest of this paper, we assume that (g2) and (g3) hold. We put $H=H^{1}\left(R^{N}\right)$. Then $H$ is a Hilbert space with norm

$$
\|u\|=\left(\int_{R^{N}}\left(|\nabla u|^{2}+|u|^{2}\right) d x\right)^{1 / 2} .
$$

The norm of the dual space $H^{-1}\left(R^{N}\right)$ of $H$ is also denoted by $\|\cdot\|$. $B_{r}$ stands for the open ball centered at 0 with radius $r$. For subsets $A, B$ of $H$ with $A \subset B$, we denote by $\operatorname{int}_{B} A$ and $\partial_{B} A$ the relative interior of $A$ in $B$ and the relative boundary of $A$ in $B$, respectively. For subsets $A, B$ of $H$, we write $A \cong B$ when $A$ and $B$ have the same homotopy type. The norm and inner product of $L^{2}\left(R^{N}\right)$ are denoted by $|\cdot|_{L^{2}}$ and $\langle\cdot, \cdot\rangle$, respectively. For each $x \in R^{N}$ and $u \in H$, we set $\tau_{x} u=u(\cdot+x)$. For each functional $F$ on $H$ and $a \in R$, we set

$$
F_{a}=\{u \in H: F(u) \leq a\} \quad \text { and } \quad \dot{F}_{a}=\{u \in H: F(u)<a\}
$$

We put

$$
\begin{aligned}
M & =\left\{u \in H \backslash\{0\}:\|u\|^{2}=\int_{R^{N}} u g(x, u) d x\right\}, \\
M^{\infty} & =\left\{u \in H \backslash\{0\}:\|u\|^{2}=\int_{R^{N}} u^{p+1} d x\right\} .
\end{aligned}
$$

From the assumption (g2), we find that for each $u \in H \backslash\{0\}$,

$$
\frac{d I(t u)}{d t}(0)=0, \quad \frac{d^{2} I(t u)}{d t^{2}}(0)=|\nabla u|_{L^{2}}^{2}+|u|_{L^{2}}^{2}-\left\langle g_{t}(x, 0) u, u\right\rangle>0,
$$

and

$$
\frac{d^{3} I(t u)}{d t^{3}}(t)=-\left\langle g_{t t}(x, t u) u^{2}, u\right\rangle<0 \quad \text { for } t>0 .
$$


Then, noting that $(d I(t u) / d t)(\lambda)=0$ if $\lambda u \in M$, we can see that there exists a positive number $\lambda_{0}(u)$ such that $I_{u}=\{\lambda u: \lambda>0\}$ intersects $M$ at exactly one point $\lambda_{0}(u) u$. Similarly, we can define a positive number $\lambda_{\infty}(u)$ by $\lambda_{\infty}(u) u \in M^{\infty}$. For simplicity, we write $\lambda_{0} u$ and $\lambda_{\infty} u$ instead of $\lambda_{0}(u) u$ and $\lambda_{\infty}(u) u$ respectively, when it is clear in the context what it means. It also follows from the definition of $M^{\infty}$ that for each $u \in M^{\infty}$,

$$
\begin{aligned}
I^{\infty}(u) & =\frac{p-1}{2(p+1)} \int_{R^{N}}\left(|\nabla u|^{2}+|u|^{2}\right) d x \\
& =\frac{p-1}{2(p+1)} \int_{R^{N}}|\nabla u|^{p+1} d x .
\end{aligned}
$$

It is known that there exists a positive radial solution $u_{\infty}$ of problem

$$
\left\{\begin{array}{l}
-\Delta u+u=|u|^{p-1} u, \quad x \in R^{N} \\
u \in H^{1}\left(R^{N}\right),
\end{array}\right.
$$

such that $c=I^{\infty}\left(u_{\infty}\right)=\min \left\{I^{\infty}(u): u \in M^{\infty}\right\}$. In [6], Kwong showed that $u_{\infty}$ is the unique positive solution up to the translation. It then follows as a direct consequence of the concentrate compactness lemma(cf. Lions [8]) that the second critical level of $I^{\infty}$ is $2 c$. That is,

Lemma 2.1. For each $0<\epsilon<c, \inf \left\{\left\|\nabla I^{\infty}(u)\right\|: u \in I_{2 c-\epsilon} \backslash \dot{I}_{c+\epsilon}\right\}>0$.

We put $c_{1}=\inf \{I(u): u \in M\}$. It then follows from the definition of $I$ and $M$ that if $u \in M$ satisfies $c_{1}=I(u)$, then $u$ is a solution of $(\mathrm{P})$. It also follows that $u$ is positive. In fact, if $u^{+}=\max \{u, 0\} \not \equiv 0$ and $u^{-}=$ $-\min \{u, 0\} \not \equiv 0$, then $u^{ \pm} \in M$ and therefore $I(u)=I\left(u^{+}\right)+I\left(u^{-}\right) \geq 2 c_{1}$. This is a contradiction. Then to find a positive solution of problem $(\mathrm{P})$, we will find a critical point of $M$ with critical level $c_{1}$. We can see from $(\mathrm{g} 3)$ that $\lim _{|x| \rightarrow \infty} I\left(u_{\infty}(\cdot+x)\right)=c$. Therefore we have that $c_{1} \leq c$. Moreover we have

Proposition 2.2. Suppose that $(g 1)$ holds with $d \leq \widetilde{d}_{0}$, where $\widetilde{d}_{0}$ is a positive number such that

$$
\delta=\inf \left\{\frac{1-d}{2}-\frac{(1+d)^{2}}{(1-d)(p+1)}: 0 \leq d \leq \widetilde{d}_{0}\right\}>0 .
$$

If $c_{1}<c$, then there exists a positive solution of problem $(P)$.

Proof. Let $u \in H$. Then by (g1), we have

$$
\begin{aligned}
I(u) & =\frac{1}{2} \int_{R^{N}}\left(|\nabla u|^{2}+|u|^{2}\right) d x-\int_{R^{N}} \int_{0}^{u(x)} g(x, t) d t d x \\
& \geq \int_{R^{N}}\left(\frac{1}{2}\left(|\nabla u|^{2}+(1-d)|u|^{2}\right)-\frac{1+d}{p+1}|u|^{p+1}\right) d x .
\end{aligned}
$$


Suppose that $u \in M$. Then again by (g1), we have

$$
\|u\|^{2}=\int_{R^{N}} u g(x, u) d x \geq \int_{R^{N}}\left(-d|u|^{2}+(1-d)|u|^{p+1}\right) d x .
$$

Combining the inequalities above, we have

$$
\begin{aligned}
I(u) \geq & \int_{R^{N}}\left(\frac{1}{2}-\frac{1+d}{(1-d)(p+1)}|\nabla u|^{2}\right. \\
& \left.+\left(\frac{1-d}{2}-\frac{(1+d)^{2}}{(1-d)(p+1)}\right)|u|^{2}\right) d x \\
\geq & \delta \int_{R^{N}}\left(|\nabla u|^{2}+|u|^{2}\right) d x .
\end{aligned}
$$

Let $\left\{u_{n}\right\} \subset M$ be a sequence such that $\lim _{n \rightarrow \infty} I\left(u_{n}\right)=c_{1}$ and $\lim _{n \rightarrow \infty} \nabla I\left(u_{n}\right)=0$. It then follows from $(2.3)$ that $\left\{u_{n}\right\}$ is bounded in $H$. Then by a parallel argument as in the proof of theorem I.2 of Lions [9], we can see that $\left\{u_{n}\right\}$ converges to $u \in H$ and $\nabla I(u)=0$ and this completes the proof.

By Proposition 2.2, it is sufficient to consider the case that $c_{1}=c$. In the sequel, we assume that $c_{1}=c$. We prove Theorem by contradiction, that is, we assume in the following that the functional $I$ does not have nontrivial critical points. Our purpose in the rest of this section is to prove the following Proposition.

Proposition 2.3. There exists a positive number $d_{0}<\widetilde{d}_{0}$ such that if $(g 1)$ holds with $d \leq d_{0}$, then for each $0<\epsilon<c$,

$$
H_{*}\left(I_{c+\epsilon}^{\infty}, I_{\epsilon}^{\infty}\right)=H_{*}\left(I_{c+\epsilon}, I_{\epsilon}\right)
$$

where $H_{*}(A, B)$ denotes the singular homology group for a pair $(A, B)$ of topological spaces (cf. Spanier [11]).

In the following we denote by $M^{0, \infty}$ and $M_{\alpha}(\alpha>0)$ the sets defined by $M^{0, \infty}=\left\{t \lambda_{0} u+(1-t) \lambda_{\infty} u: u \in H \backslash\{0\}, t \in[0,1], \lambda_{0} u \in M, \lambda_{\infty} u \in M^{\infty}\right\}$

and

$$
M_{\alpha}=\left\{(1+\tau) u: u \in M^{0, \infty}, \tau \in(-R(u), R(u))\right\}
$$

where

$$
\begin{aligned}
& R(u)=\sup \left\{t>0: \max \left\{\frac{I(u)}{I((1+\tau) u)}, \frac{I^{\infty}((1+\tau) u)}{I^{\infty}(u)}\right\}<1+\alpha\right. \\
& \quad \text { for all } \tau \in[-t, t]\} .
\end{aligned}
$$

From the definition, $M^{\infty}, M \subset M^{0, \infty}$ and $M_{\alpha}$ is an open neighborhood of $M^{0, \infty}$. 
Lemma 2.4. There exist positive numbers $d_{1}$ and $\alpha_{0}$ such that if (g1) holds with $d \leq d_{1}$, then for each positive number $\alpha<\alpha_{0}$,

$$
\begin{aligned}
& I_{(7 / 6) c}^{\infty} \subset I_{(4 / 3) c} \cup\left(M_{\alpha}\right)^{c}, \\
& I_{(4 / 3) c} \subset I_{(5 / 3) c}^{\infty} \cup\left(M_{\alpha}\right)^{c}, \\
& I_{(5 / 3) c}^{\infty} \subset I_{(11 / 6) c} \cup\left(M_{\alpha}\right)^{c} .
\end{aligned}
$$

Proof. The assertions (1), (2) and (3) can be proved by parallel arguments. We give only the proof of $(2)$. Let $d_{1}>0$ such that

$$
\begin{aligned}
\frac{4}{5}<\rho & =\min \left\{\left(\frac{(1-d)^{2}}{2(1+d)}-\frac{1+d}{p+1}\right) \frac{2(p+1)}{p-1}\right. \\
& \left.\left(\frac{1-d}{1+d}\right)^{2 /(p-1)}\left(\frac{2(p+1)}{p-1}\right)\left(\frac{1-d}{2}-\frac{(1+d)}{p+1}\right)\right\}, \text { for } 0 \leq d \leq d_{0} .
\end{aligned}
$$

We assume that (g1) holds with $d \leq d_{1}$. Fix $u \in H \backslash\{0\}$. Then we have from the definitions of $M$ and $M^{\infty}$ that

(2.6) $\left\|\lambda_{0} u\right\|^{2}=\int_{R^{N}} \lambda_{0} u g\left(x, \lambda_{0} u\right) d x$ and $\left\|\lambda_{\infty} u\right\|^{2}=\int_{R^{N}}\left|\lambda_{\infty} u\right|^{p+1} d x$.

By (g1) and (2.6), we have

$$
\begin{aligned}
\frac{1-d}{1+d} \int_{R^{N}}\left|\lambda_{0} u\right|^{p+1} d x & \leq \frac{1}{1+d} \int_{R^{N}}\left(\lambda_{0} u g\left(x, \lambda_{0} u\right)+d\left|\lambda_{0} u\right|^{2}\right) d x \\
& \leq \frac{1}{1+d} \int_{R^{N}}\left(\left|\nabla \lambda_{0} u\right|^{2} d x+(1+d)\left|\lambda_{0} u\right|^{2}\right) d x \\
& \leq \int_{R^{N}}\left(\left|\nabla \lambda_{0} u\right|^{2} d x+\left|\lambda_{0} u\right|^{2}\right) d x \\
& \leq \frac{1}{1-d} \int_{R^{N}}\left(\left|\nabla \lambda_{0} u\right|^{2} d x+(1-d)\left|\lambda_{0} u\right|^{2}\right) d x \\
& \leq \frac{1}{1-d} \int_{R^{N}}\left(\lambda_{0} u g\left(x, \lambda_{0} u\right)-d\left|\lambda_{0} u\right|^{2}\right) d x \\
& \leq \frac{1+d}{1-d} \int_{R^{N}}\left|\lambda_{0} u\right|^{p+1} d x .
\end{aligned}
$$

That is, we have

$$
\begin{aligned}
\frac{1-d}{1+d} \int_{R^{N}}\left|\lambda_{0} u\right|^{p+1} d x & \leq \int_{R^{N}}\left(\left|\nabla \lambda_{0} u\right|^{2} d x+\left|\lambda_{0} u\right|^{2}\right) d x \\
& \leq \frac{1+d}{1-d} \int_{R^{N}}\left|\lambda_{0} u\right|^{p+1} d x .
\end{aligned}
$$


We find from the second equality of (2.6) and (2.7) that

$$
\frac{1-d}{1+d} \lambda_{0}^{p-1} \leq \lambda_{\infty}^{p-1} \leq \frac{1+d}{1-d} \lambda_{0}^{p-1}
$$

To prove the assertion, we will show that for $0<\alpha<\alpha_{0}$,

$$
I_{(4 / 3) c} \cap M_{\alpha} \subset I_{(5 / 3) c}^{\infty}
$$

Now let $u \in M^{0, \infty}$. From the definition of $M^{0, \infty}$, we have that $\lambda_{0} \leq 1 \leq \lambda_{\infty}$ or $\lambda_{\infty} \leq 1 \leq \lambda_{0}$ holds. We first consider the case that $\lambda_{\infty} \leq 1 \leq \lambda_{0}$. Since $\lambda_{\infty} \leq 1$, we have that

$$
\|u\|^{2}=\int_{R^{N}}\left(|\nabla u|^{2}+|u|^{2}\right) d x \leq \int_{R^{N}}|u|^{p+1} d x .
$$

Then we find that

$$
I^{\infty}(u) \leq \frac{p-1}{2(p+1)} \int_{R^{N}}|u|^{p+1} d x .
$$

On the other hand, recalling that the second equality of (2.6) holds, we obtain from (g1), (2.9) and (2.8) that

$$
\begin{aligned}
I(u) & \geq \frac{1-d}{2} \int_{R^{N}}\left(|\nabla u|^{2}+|u|^{2}\right) d x-\frac{1+d}{p+1} \int_{R^{N}}|u|^{p+1} d x \\
& =\left(\frac{1-d}{2} \lambda_{\infty}^{p-1}-\frac{1+d}{p+1}\right) \int_{R^{N}}|u|^{p+1} d x \\
& \geq\left(\frac{(1-d)^{2}}{2(1+d)}-\frac{1+d}{p+1}\right) \frac{2(p+1)}{p-1} I^{\infty}(u) \\
& \geq \rho I^{\infty}(u) .
\end{aligned}
$$

We choose a positive number $\alpha_{1}<1$ such that $4 / 5<\rho /\left(1+\alpha_{1}\right)^{2}$. Now suppose that $(1+\tau) u \in M_{\alpha_{1}}, \tau \in R$. Then, by (2.10), we have

$$
\begin{aligned}
I((1+\tau) u) \geq\left(1 /\left(1+\alpha_{1}\right)\right) I(u) & \geq\left(\rho /\left(1+\alpha_{1}\right)\right) I^{\infty}(u) \\
& \geq\left(\rho /\left(1+\alpha_{1}\right)^{2}\right) I^{\infty}((1+\tau) u) .
\end{aligned}
$$

Assume that $(1+\tau) u \in I_{(4 / 3) c}$. Then it follows from the inequalities above that

$$
I^{\infty}((1+\tau) u) \leq(4 / 3) c\left(1+\alpha_{1}\right)^{2} / \rho \leq(5 / 3) c .
$$

We next assume that $\lambda_{0} \leq 1 \leq \lambda_{\infty}$. Then by (2.2),

$$
I^{\infty}(u) \leq I^{\infty}\left(\lambda_{\infty} u\right)=\frac{p-1}{2(p+1)} \lambda_{\infty}^{2} \int_{R^{N}}\left(|\nabla u|^{2}+|u|^{2}\right) d x
$$


On the other hand, we have by (2.8) that

$$
\lambda_{\infty} \leq\left(\frac{1+d}{1-d}\right)^{1 /(p-1)} .
$$

Then, noting that $\lambda_{\infty}^{-(p-1)} \leq 1$, we have from (g1) and (2.11) that

$$
\begin{aligned}
I(u) & \geq \frac{1-d}{2} \int_{R^{N}}\left(|\nabla u|^{2}+|u|^{2}\right) d x-\frac{1+d}{p+1} \int_{R^{N}}|u|^{p+1} d x \\
& \geq\left(\frac{1-d}{2}-\frac{1+d}{p+1} \lambda_{\infty}^{-(p-1)}\right) \int_{R^{N}}\left(|\nabla u|^{2}+|u|^{2}\right) d x \\
& =\lambda_{\infty}^{-2} \frac{2(p+1)}{p-1}\left(\frac{1-d}{2}-\lambda_{\infty}^{-(p-1)} \frac{(1+d)}{p+1}\right) I^{\infty}(u) \\
& \geq\left(\frac{1-d}{1+d}\right)^{2 /(p-1)} \frac{2(p+1)}{p-1}\left(\frac{1-d}{2}-\frac{(1+d)}{p+1}\right) I^{\infty}(u) \\
& \geq \rho I^{\infty}(u) .
\end{aligned}
$$

Then we have that there exists $\alpha_{2}>0$ such that for all $u \in M_{\alpha_{2}}$ with $I(u) \leq(4 / 3) c, I^{\infty}(u) \leq(5 / 3) c$. Thus we obtain that the assertion holds with $\alpha_{0}=\min \left\{\alpha_{1}, \alpha_{2}\right\}$.

Throughout the rest of this section we fix the positive number $\alpha<\alpha_{0}$.

Lemma 2.5. There exists a continuous mapping $\gamma_{1}:[0,1] \times\left(I_{(11 / 6) c} \cup\right.$ $\left.M_{\alpha}^{c}\right) \rightarrow I_{(11 / 6) c} \cup M_{\alpha}^{c}$ such hat

(i) $\gamma_{1}(0, x)=x \quad$ for all $x \in I_{(11 / 6) c} \cup M_{\alpha}^{c}$,

(ii) $\gamma_{1}(t, x)=x \quad$ for all $(t, x) \in[0,1] \times\left(I_{(4 / 3) c} \cup M_{\alpha}^{c}\right)$,

(iii) $I\left(\gamma_{1}(t, x)\right) \leq I\left(\gamma_{1}(0, x)\right) \quad$ for all $(t, x) \in[0,1] \times\left(I_{(11 / 6) c} \cup M_{\alpha}^{c}\right)$,

(iv) $\gamma_{1}\left(1, I_{(11 / 6) c} \cup M_{\alpha}^{c}\right) \subset I_{(4 / 3) c} \cup M_{\alpha}^{c}$.

Proof. We set

$$
M_{o}=\{\lambda u: u \in M, \lambda>1\} \quad \text { and } \quad M_{i}=\{\lambda u: u \in M, \lambda<1\} .
$$

Let $U$ be an open set such that

$$
\left(M_{\alpha}\right)^{c} \subset U \quad \text { and } \quad U \cap M_{\alpha / 2}=\phi .
$$

Then since $M \subset M_{\alpha / 2}$, we can see that

$$
\langle\nabla I(v), v\rangle>0 \quad \text { on } M_{i} \cap U \quad \text { and } \quad\langle\nabla I(v), v\rangle<0 \quad \text { on } M_{o} \cap U \text {. }
$$

Then by arguing standard way (cf. Lemma 1.6 of Rabinowitz [10]), we can construct a pseudo-gradient vector field $\widetilde{V}$ associated with $\nabla I$ such that
(a) $\|\widetilde{V}(u)\| \leq 2\|\nabla I(u)\|, \quad$ for $u \in H$;
(b) $\langle\nabla I(u), \widetilde{V}(u)\rangle \geq\|\nabla I(u)\|^{2}, \quad$ for $u \in H$;
(c) $\langle\widetilde{V}(v), v\rangle>0 \quad$ on $M_{i} \cap U$;
(d) $\langle\widetilde{V}(v), v\rangle<0 \quad$ on $M_{o} \cap U$. 
We put

$$
\begin{array}{ll}
h_{1}(v)=\left\|v-M_{\alpha}^{c}\right\| /\left(\left\|v-U^{c}\right\|+\left\|v-M_{\alpha}^{c}\right\|\right) & \text { for } v \in H, \\
h_{2}(v)=\left\|v-U^{c}\right\| /\left(\left\|v-U^{c}\right\|+\left\|v-M_{\alpha}^{c}\right\|\right) & \text { for } v \in H
\end{array}
$$

and

$$
V(v)=h_{1}(v) \widetilde{V}(v)+h_{2}(v) \operatorname{sgn}(\langle\widetilde{V}(v), v\rangle) v \quad \text { for } v \in H
$$

Then $V$ is Lipschitz continuous on $I_{(11 / 6) c} \cup\left(M_{\alpha}\right)^{c}$. Consider the ordinary differential equation

$$
\frac{d \eta}{d t}=-V(\eta), \quad \eta(0, v)=v \quad \text { for } v \in I_{(11 / 6) c} \cup\left(M_{\alpha}\right)^{c} .
$$

The solution $\eta: R^{+} \times H \rightarrow H$ defines a semiflow on $H$. It follows from the definition of $V$ that $\eta(t, v) \in\left(M_{\alpha}\right)^{c}$ for $(t, v) \in[0, \infty) \times\left(M_{\alpha}\right)^{c}$. In fact, if $v \in\left(M_{\alpha}\right)^{c}$, then for each $t>0, \eta(t, v)=\lambda_{t} v$, where $\lambda_{t} \in R$ such that $\lambda_{t} v \in\left(M_{\alpha}\right)^{c}$. We also have from (a)-(c) and (2.13) that $\langle V(v), \nabla I(v)\rangle>0$ on $U \cup I_{(11 / 6) c}$ and then

$$
I(\eta(t, v))<I(\eta(s, v)) \quad \text { for } t>s \text { and } v \in U \cup I_{(11 / 6) c} .
$$

Thus we find that $\eta(t, v) \in I_{(11 / 6) c} \cup\left(M_{\alpha}\right)^{c}$ for $(t, v) \in[0, \infty) \times I_{(11 / 6) c} \cup$ $\left(M_{\alpha}\right)^{c}$. It follows from Lemma 2.1 that

$$
\inf \left\{\|\nabla I(u)\|: u \in I_{(11 / 6) c} \backslash I_{(4 / 3) c}\right\}>0 .
$$

Then we have

$$
\inf \left\{\|V(u)\|: u \in\left(U \cup I_{(11 / 6) c}\right) \backslash I_{(4 / 3) c}\right\}>0 .
$$

Therefore, there exists $T>0$ such that

$$
\begin{aligned}
\eta(t, v) \in \operatorname{int}\left(I_{(4 / 3) c} \cup\left(M_{\alpha}\right)^{c}\right) & \text { for all } t>T \\
& \text { and all } v \in I_{(11 / 6) c} \cup\left(M_{\alpha}\right)^{c} .
\end{aligned}
$$

Here we put

$$
\gamma(t, v)=\eta\left(t_{v} \cdot t, v\right) \quad \text { for }(t, v) \in[0,1] \times I_{(11 / 6) c} \cup\left(M_{\alpha}\right)^{c},
$$

where

$$
t_{v}=\inf \left\{t \geq 0: \eta(t, v) \in I_{(4 / 3) c} \cup\left(M_{\alpha}\right)^{c}\right\} \quad \text { for } v \in I_{(11 / 6) c} \cup\left(M_{\alpha}\right)^{c} .
$$

Then, by (2.15), we have $\gamma_{1}:[0,1] \times I_{(11 / 6) c} \cup\left(M_{\alpha}\right)^{c} \rightarrow I_{(11 / 6) c} \cup\left(M_{\alpha}\right)^{c}$ satisfying the desired properties.

By a parallel argument as in the proof of Lemma 2.5, we have 
Lemma 2.6. There exists a continuous mapping $\gamma_{2}:[0,1] \times I_{(5 / 3) c}^{\infty} \cup M_{\alpha}^{c} \rightarrow$ $I_{(5 / 3) c}^{\infty} \cup M_{\alpha}^{c}$ such that

$$
\begin{aligned}
& \text { (v) } \gamma_{2}(0, x)=x \\
& \text { for all } x \in I_{(5 / 3) c}^{\infty} \cup M_{\alpha}^{c} \text {; } \\
& \text { (vi) } \gamma_{2}(t, x)=x \\
& \text { for all }(t, x) \in[0,1] \times\left(I_{(7 / 6) c}^{\infty} \cup M_{\alpha}^{c}\right) \text {; } \\
& \text { (vii) } I^{\infty}\left(\gamma_{2}(t, x)\right) \leq I^{\infty}\left(\gamma_{2}(0, x)\right) \quad \text { for all }(t, x) \in[0,1] \times\left(I_{(5 / 3) c}^{\infty} \cup M_{\alpha}^{c}\right) \text {; } \\
& \text { (viii) } \gamma_{2}\left(1, I_{(5 / 3) c}^{\infty} \cup M_{\alpha}^{c}\right) \subset I_{(7 / 6) c}^{\infty} \cup M_{\alpha}^{c} \text {. }
\end{aligned}
$$

Lemma 2.7. For each $0<\epsilon<c, I_{\epsilon}^{\infty}$ and $I_{\epsilon}$ have the same homotopy type.

Proof. Let $0<\epsilon<c$. Then we have by (2.1) that there exist continuous mappings $t_{1}: H \backslash\{0\} \rightarrow R^{+}$and $t_{2}: H \backslash\{0\} \rightarrow R^{+}$such that for each $u \in H \backslash\{0\}, t_{1}(u)<t_{2}(u)$ and

$$
\{I(t u): t \geq 0\} \cap I_{\epsilon}=\left\{t u: t \in\left[0, t_{1}(u)\right] \cup\left[t_{2}(u), \infty\right)\right\}
$$

Similarly, there exist continuous mappings $t_{1}^{\infty}: H \backslash\{0\} \rightarrow R^{+}$and $t_{2}^{\infty}$ : $H \backslash\{0\} \rightarrow R^{+}$such that for each $u \in H \backslash\{0\}, t_{1}^{\infty}(u)<t_{2}^{\infty}(u)$ and

$$
\left\{I^{\infty}(t u): t \geq 0\right\} \cap I_{\epsilon}^{\infty}=\left\{t u: t \in\left[0, t_{1}^{\infty}(u)\right] \cup\left[t_{2}^{\infty}(u), \infty\right)\right\} .
$$

Then we find that $I_{\epsilon}^{\infty}$ and $I_{\epsilon}$ have the same homotopy type.

We can now prove Proposition 2.3.

Proof of Proposition 2.3. Let $0<\epsilon<c$. Then $I_{c+\epsilon}^{\infty}$ and $I_{c+\epsilon}$ have the same homotopy types as $I_{(7 / 6) c}^{\infty}$ and $I_{(7 / 6) c}$, respectively. We also have that $I_{\epsilon}^{\infty}$ and $I_{\epsilon}$ have the same homotopy types with as $I_{(1 / 3) c}^{\infty}$ and $I_{(1 / 3) c}$, respectively. Then to prove the assertion, it is sufficient to show that

$$
H_{*}\left(I_{(7 / 6) c}^{\infty}, I_{(1 / 3) c}^{\infty}\right) \cong H_{*}\left(I_{(7 / 6) c}, I_{(1 / 3) c}\right)
$$

We first define a mapping $\widetilde{\gamma}:[0,1] \times\left(I_{(11 / 6) c} \cup\left(M_{\alpha}\right)^{c}\right) \rightarrow I_{(11 / 6) c} \cup\left(M_{\alpha}\right)^{c}$ by

$$
\widetilde{\gamma}(t, u)= \begin{cases}\gamma_{1}(2 t, u), & \text { for } t \in[0,1 / 2], \\ \gamma_{2}\left(2(t-1 / 2), \gamma_{1}(1, u)\right), & \text { for } t \in(1 / 2,1] .\end{cases}
$$

Then from (iii), we have that

$$
\widetilde{\gamma}(t, u) \in I_{(11 / 6) c} \cup\left(M_{\alpha}\right)^{c}
$$

for $(t, u) \in[0,1 / 2] \times\left(I_{(11 / 6) c} \cup\left(M_{\alpha}\right)^{c}\right)$. On the other hand, we have, by combining (iv) and (vii) with (3) of Lemma 2.4, that (2.16) holds for $(t, u) \in$ $[1 / 2,1] \times\left(I_{(11 / 6) c} \cup\left(M_{\alpha}\right)^{c}\right)$. Thus we have that $\widetilde{\gamma}$ is well defined and a strong deformation retraction from $I_{(11 / 6) c} \cup\left(M_{\alpha}\right)^{c}$ onto $I_{(7 / 6) c}^{\infty} \cup\left(M_{\alpha}\right)^{c}$. We next 
define a mapping $\gamma_{3}:[0,1] \times\left(I_{(7 / 6) c}^{\infty} \cup M_{\alpha}^{c}\right) \rightarrow I_{(7 / 6) c}^{\infty}$. For each $u \in\left(M_{\alpha}\right)^{c}$ with $I^{\infty}(u)>(7 / 6) c$, we set

$$
\begin{aligned}
& \tau_{u}^{+}=\min \left\{\tau>1: I^{\infty}(\tau u) \leq(7 / 6) c\right\}, \\
& \tau_{u}^{-}=\max \left\{\tau<1: I^{\infty}(\tau u) \leq(7 / 6) c\right\}, \\
& M_{o}^{\infty}=\left\{\lambda u: u \in M^{\infty}, \lambda>1\right\}
\end{aligned}
$$

and

$$
M_{i}^{\infty}=\left\{\lambda u: u \in M^{\infty}, \lambda<1\right\} .
$$

Then we put

$$
\gamma_{3}(t, x)= \begin{cases}t \tau_{u}^{+} u+(1-t) u & \text { if } u \in M_{o}^{\infty} \backslash\left(I_{(7 / 6) c}^{\infty} \cup M_{\alpha}\right), \\ t \tau_{u}^{-} u+(1-t) u & \text { if } u \in M_{i}^{\infty} \backslash\left(I_{(7 / 6) c}^{\infty} \cup M_{\alpha}\right), \\ u & \text { if } u \in I_{(7 / 6) c}^{\infty} .\end{cases}
$$

It then easy to see that $\gamma_{3}$ is a strong deformation retraction from $I_{(7 / 6) c}^{\infty} \cup$ $\left(M_{\alpha}\right)^{c}$ to $I_{(7 / 6) c}^{\infty}$. Therefore we obtain that $I_{(7 / 6) c}^{\infty}$ is a strong deformation retract of $I_{(11 / 6) c} \cup\left(M_{\alpha}\right)^{c}$. It then follows that

$$
H_{*}\left(I_{(7 / 6) c}^{\infty}, I_{(1 / 3) \epsilon}^{\infty}\right)=H_{*}\left(I_{(11 / 6) c} \cup\left(M_{\alpha}\right)^{c}, I_{(1 / 3) \epsilon}^{\infty}\right) .
$$

Then by Lemma 2.7,

$$
H_{*}\left(I_{(11 / 6) c} \cup\left(M_{\alpha}\right)^{c}, I_{(1 / 3) \epsilon}^{\infty}\right)=H_{*}\left(I_{(11 / 6) c} \cup\left(M_{\alpha}\right)^{c}, I_{(1 / 3) \epsilon}\right) .
$$

On the other hand, we can see by a parallel argument as above that $I_{(7 / 6) c}$ is a strong deformation retract of $I_{(11 / 6) c} \cup\left(M_{\alpha}\right)^{c}$. Then from (2.17) and (2.18), we have $H_{*}\left(I_{(7 / 6) c}^{\infty}, I_{(1 / 3) c}^{\infty}\right) \cong H_{*}\left(I_{(7 / 6) c}, I_{(1 / 3) c}\right)$, which completes the proof.

\section{Proof of the Theorem}

We start with the following proposition.

Proposition 3.1. For each positive number $\epsilon<c$,

$$
H_{q}\left(I_{c+\epsilon}^{\infty}, I_{\epsilon}^{\infty}\right)= \begin{cases}2 & \text { if } q=0, \\ 0 & \text { if } q \neq 0 .\end{cases}
$$

Proposition 3.1 was proved in [6]. For completeness, we give the proof of it in the appendix. We next consider a triple $(U, K, \epsilon) \subset H \times H \times R^{+}$ satisfying the following conditions:

(1) $U \cap(-U)=\phi$;

(2) $\left\{\tau_{x} u_{\infty}:|x| \geq r\right\} \subset$ int $K$ for some $r>0$;

(3) $\operatorname{cl}\left(I_{c+\epsilon} \cap K\right) \subset \operatorname{int}_{I_{c+\epsilon}}\left(I_{c+\epsilon} \cap U\right)$;

(4) $I_{\epsilon}$ is a strong deformation retract of $I_{c+\epsilon} \backslash(K \cup(-K))$;

(5) $H_{N-1}\left(I_{c+\epsilon} \cap U\right)=1, \quad H_{1}\left(I_{c+\epsilon} \cap U\right)=0$;

(6) $H_{N-1}\left(\left(I_{c+\epsilon} \cap U\right) \backslash K\right)=2$ or $H_{0}\left(\left(I_{c+\epsilon} \cap U\right) \backslash K\right)=1$ holds. 
Proposition 3.2. There exists a triple $(U, K, \epsilon) \subset H \times H \times R^{+}$which satisfies $(1)-(6)$.

The proof of Proposition 3.2 is given in Section 4 .

Lemma 3.3. Suppose that there exist a triple $(U, K, \epsilon) \subset H \times H \times R^{+}$ satisfying $(1)-(6)$. Suppose, in addition, that $H_{N-1}\left(\left(I_{c+\epsilon} \cap U\right) \backslash K\right) \geq 2$. Then $H_{N}\left(I_{c+\epsilon}, I_{\epsilon}\right) \geq 2$.

Proof. We put $\widetilde{K}=K \cup(-K)$. Since $I_{\epsilon}$ is a strong deformation retract of $I_{c+\epsilon} \backslash \widetilde{K}$, we find that

$$
H_{q}\left(I_{c+\epsilon} \backslash \widetilde{K}, I_{\epsilon}\right) \cong H_{q}\left(I_{\epsilon}, I_{\epsilon}\right) \cong 0
$$

Then we have from the exactness of the singular homology groups of the triple $\left(I_{c+\epsilon}, I_{c+\epsilon} \backslash \widetilde{K}, I_{\epsilon}\right)$ that

$$
0 \rightarrow H_{q}\left(I_{c+\epsilon}, I_{\epsilon}\right) \rightarrow H_{q}\left(I_{c+\epsilon}, I_{c+\epsilon} \backslash \widetilde{K}\right) \rightarrow 0 .
$$

That is,

$$
H_{q}\left(I_{c+\epsilon}, I_{\epsilon}\right) \cong H_{q}\left(I_{c+\epsilon}, I_{c+\epsilon} \backslash \widetilde{K}\right) .
$$

From (1) and (3), we find

$$
H_{q}\left(I_{c+\epsilon}, I_{c+\epsilon} \backslash \widetilde{K}\right) \cong H_{q}(W, W \backslash K) \oplus H_{q}(-W,(-W) \backslash(-K)),
$$

where $W=I_{c+\epsilon} \cap U$. Then since $H_{N-1}(W \backslash K) \geq 2$, we have from (5) and the exactness of the sequence

$$
\begin{aligned}
\rightarrow H_{q}(W, W \backslash K) & \rightarrow H_{q-1}(W \backslash K) \\
& \rightarrow H_{q-1}(W) \rightarrow H_{q-1}(W, W \backslash K) \rightarrow,
\end{aligned}
$$

with $q=N$, that $H_{N}\left(I_{c+\epsilon}, I_{\epsilon}\right) \cong H_{N}(W, W \backslash K) \oplus H_{N}(W, W \backslash K) \geq 2$.

Lemma 3.4. Suppose that $(U, K, \epsilon) \subset H \times H \times R^{+}$satisfies (1) - (6). Suppose in addition that $H_{0}\left(I_{c+\epsilon} \cap U\right)=H_{0}\left(\left(I_{c+\epsilon} \cap U\right) \backslash K\right)=1$. Then $H_{1}\left(I_{c+\epsilon}, I_{\epsilon}\right)=0$ or $H_{0}\left(I_{c+\epsilon}, I_{\epsilon}\right)=2$ holds.

Proof. From the argument in the proof of Proposition 3.2, we have

$$
H_{1}\left(I_{c+\epsilon}, I_{\epsilon}\right) \cong H_{1}\left(I_{c+\epsilon} \cap U,\left(I_{c+\epsilon} \cap U\right) \backslash K\right) \oplus H_{1}\left(I_{c+\epsilon} \cap U,\left(I_{c+\epsilon} \cap U\right) \backslash K\right) .
$$

Then since $H_{1}\left(I_{c+\epsilon} \cap U\right)=0$ and $H_{0}\left(I_{c+\epsilon} \cap U\right)=H_{0}\left(\left(I_{c+\epsilon} \cap U\right) \backslash K\right)=1$, the assertion follows from the exactness of the sequence (3.1) with $q=1$.

We can now prove the Theorem.

Proof of the Theorem. Let $(U, K, \epsilon)$ satisfy $(1)-(6)$. We have by Proposition 2.3 and Proposition 3.1 that $H_{1}\left(I_{c+\epsilon}, I_{\epsilon}\right)=2$ and $H_{q}\left(I_{c+\epsilon}, I_{\epsilon}\right)=$ 0 for $q \neq 1$. Now suppose that $\left(I_{c+\epsilon} \cap U\right) \backslash K$ is disconnected. Then since $H_{0}\left(\left(I_{c+\epsilon} \cap U\right) \backslash K\right) \geq 2$, we find by $(6)$ that $H_{N-1}\left(I_{c+\epsilon}, I_{\epsilon}\right)=2$. This is a contradiction. On the other hand, if $U \backslash K$ is connected, then $H_{0}\left(\left(I_{c+\epsilon} \cup U\right) \backslash K\right)=1$. Then by Lemma 3.4, we have $H_{1}\left(I_{c+\epsilon}, I_{\epsilon}\right)=0$ or $H_{0}\left(I_{c+\epsilon}, I_{\epsilon}\right)=2$. This is a contradiction. Thus we obtain that there exists a positive solution of $(\mathrm{P})$. 


\section{Proof of Proposition 3.2}

We shall construct a triple $(U, K, \epsilon)$ satisfying $(1)-(6)$. First we state the following lemma.

Lemma 4.1. If $0<\epsilon<c<d<2 c$ and $\left\{u_{n}\right\} \subset I_{d} \backslash I_{\epsilon}$ is a sequence such that $\nabla I\left(u_{n}\right) \rightarrow 0$, then $u_{n} \rightarrow \tau_{x_{n}} u_{\infty}$ where $\left\{x_{n}\right\} \subset R^{N}$ with $\lim _{n \rightarrow \infty}\left|x_{n}\right|=$ $\infty$.

Since we are assuming that $I$ has no critical point in $\dot{I}_{2 c} \backslash I_{c}$, the assertion of Lemma 4.1 is a direct consequence of the arguments in $[8,9]$. Thus, we omit the proof (cf. also [3]).

We fix a positive number $\rho<1$. Recalling that the mappings $t \rightarrow$ $I^{\infty}\left(( \pm t+1) u_{\infty}\right)$ are decreasing as $\mathrm{t}$ varies from 0 to \pm 1 , we have $I_{c}^{\infty} \cap$ $\left\{t u_{\infty}: t \in[-\rho+1, \rho+1]\right\}=\left\{u_{\infty}\right\}$. Then we can choose positive numbers $r_{0}$ and $\delta$ such that

$$
\left\{t v: t \in[-\rho+1,-\rho / 2+1] \cup[\rho / 2+1, \rho+1], v \in S_{0}\right\} \subset I_{c-\delta}^{\infty}
$$

where $S_{0}=\left(u_{\infty}+B_{r_{0}}\right) \cap M^{\infty}$. We note that $S_{0}$ is a contractible neighborhood of $u_{\infty}$ in $M^{\infty}$. We may choose $r_{0}$ so small that

$$
S_{0} \subset I_{(4 / 3) c}^{\infty}
$$

Next, we fix a contractible neighborhood $\widetilde{S}_{0}$ of $u_{\infty}$ in $M^{\infty}$ such that $\widetilde{S}_{0} \subset$ int $_{M \infty} S_{0}$. We put

$$
\begin{aligned}
& D_{0}=\left\{\tau_{x} v: v \in S_{0}, x \in R^{N} \text { with }|x| \geq R_{0}\right\}, \\
& \widetilde{D}_{0}=\left\{\tau_{x} v: v \in \widetilde{S}_{0}, x \in R^{N} \text { with }|x| \geq 2 R_{0}\right\},
\end{aligned}
$$

where $R_{0}$ is a positive number. Then $\widetilde{D}_{0} \subset D_{0} \subset M^{\infty}$. Now we define subsets $U, K$ of $H$ by

$$
\begin{aligned}
& U=\left\{t v: t \in[-\rho+1, \rho+1], v \in D_{0}\right\} \\
& K=\left\{t v: t \in[-\rho / 2+1, \rho / 2+1], v \in \widetilde{D}_{0}\right\} .
\end{aligned}
$$

Since $\left\{\tau_{x} u_{\infty}: x \in R^{N}\right\} \cap\left\{\tau_{x}\left(-u_{\infty}\right): x \in R^{N}\right\}=\phi$, by choosing $r_{0}$ and $\rho$ sufficiently small, we have that $U \cap(-U)=\phi$. That is, (1) holds. Since (4.1) holds and $\lim _{|x| \rightarrow \infty} I\left(\tau_{x} u_{\infty}\right)=c$, we can choose $R_{0}$ so large that

$$
\left\{t v: t \in[-\rho+1,-\rho / 2+1] \cup[\rho / 2+1, \rho+1], v \in D_{0}\right\} \subset I_{c} .
$$

We also have by (4.2) that $R_{0}$ can be chosen so large that $U \subset I_{(6 / 5) c}$. It follows from the defintion of $U$ and $K$ that

$$
\left\{\tau_{x} u_{\infty}:|x| \geq 3 R_{0}\right\} \subset \operatorname{int} K \subset K \subset \operatorname{int} U
$$


That is, (2) holds with $r=3 R_{0}$. From the definition, it is obvious that (3) holds. As a direct consequence of (3) of Lemma 4.1 and (4.5), we have

$$
\inf \left\{\|\nabla I(v)\|: \quad v \in I_{d} \backslash\left(I_{\epsilon} \cup K \cup(-K)\right)\right\}>0
$$

for all $0<\epsilon<c<d<2 c$. Then by deformation lemma(cf. [3]), there exists $\epsilon_{0}>0$ such that for each $0<\epsilon<\epsilon_{0}, I_{\epsilon}$ is a strong deformation retract of $I_{c+\epsilon} \backslash(K \cup(-K))$. That is, (4) holds for all $0<\epsilon<\epsilon_{0}$.

We will see that there exists $0<\epsilon<\epsilon_{0}$ such that $(U, K, \epsilon)$ satisifes (5) and (6). Here we note that

$$
c_{2}=\inf \left\{I\left(\lambda_{0}(v) v\right): v \in D_{0} \backslash \widetilde{D}_{0}\right\}>c_{1}
$$

In fact, if $c_{2}=c_{1}$, there exists a sequence $\left\{u_{n}\right\} \subset M$ such that $u_{n}=$ $\lambda_{0}\left(v_{n}\right) v_{n}, v_{n} \in D_{0} \backslash \widetilde{D}_{0}$ for each $n \geq 1$ and that $\lim _{n \rightarrow \infty} I\left(u_{n}\right)=c$. This implies that $\nabla I\left(u_{n}\right) \rightarrow 0$ and then by Lemma $4.1, u_{n} \rightarrow \tau_{x_{n}} u_{\infty}$, where $\left\{x_{n}\right\} \subset R^{N}$ with $\lim \left|x_{n}\right|=\infty$. This implies that $v_{n} \rightarrow \tau_{x_{n}} u_{\infty}$ and this contradicts to the definition of $\left\{v_{n}\right\}$. Here we choose a positive number $\epsilon$ such that $\epsilon<c_{2}-c$. Here we define subsets of $M$ and $H$. Noting that

$$
\lim _{|x| \rightarrow \infty} I\left(\tau_{x} u_{\infty}\right)=c
$$

We can choose contractible neighborhoods $S_{1}, S_{2}$ of $u_{\infty}$ in $M^{\infty}$ and positive numbers $R_{1}, R_{2}$ such that $S_{2} \subset \operatorname{int}_{M \infty} S_{1} \subset S_{0}, R_{1}<R_{2}$ and

$$
U_{i}=\left\{t \tau_{x} v: t \in[-\rho+1, \rho+1],|x| \geq R_{i}, v \in S_{i}\right\} \subset I_{c+\epsilon} .
$$

We also set

$$
\begin{aligned}
& U_{1,+}=\left\{t v: t \in[-\rho+1,-\rho / 2+1], v \in D_{0}\right\}, \\
& U_{1,-}=\left\{t v: t \in[\rho / 2+1, \rho+1], v \in D_{0}\right\}
\end{aligned}
$$

and

$$
\begin{aligned}
& U_{2,+}=\left\{t v: t \in[-\rho+1,-\rho / 4+1], v \in D_{0}\right\}, \\
& U_{2,-}=\left\{t v: t \in[\rho / 4+1, \rho+1], v \in D_{0}\right\} .
\end{aligned}
$$

Then from the definitions above and (4.2), we have that

$$
\widetilde{U}_{2}=U_{2} \cup U_{2,+} \cup U_{2,-} \subset \widetilde{U}_{1}=U_{1} \cup U_{1,+} \cup U_{1,-} \subset I_{c+\epsilon},
$$

and

$$
\widetilde{U}_{1} \cong \widetilde{U}_{2} \cong\left\{\tau_{x} u_{\infty}:|x| \geq R_{1}\right\} \cong S^{N-1}
$$

Then we have that (5) holds, as a direct consequence of the following lemma 4.5 . 
Lemma 4.2. $\widetilde{U}_{1}$ is a deformation reatract of $I_{c+\epsilon} \cap U$.

Proof. To prove the assertion it is sufficient to show the existence of a semiflow $\eta:[0, \infty) \times\left(I_{c+\epsilon} \cap U\right) \rightarrow I_{c+\epsilon} \cap U$ such that for each $v \in I_{c+\epsilon} \cap U$, there exists $t_{v} \geq 0$ satsifying $\eta(t, v) \in i n t_{I_{c+\epsilon} \cap U} \widetilde{U}_{1}$ for all $t \geq t_{v}$. In fact, if there exists such a semiflow, we can construct a strong deformation retraction as in the proof of Lemma 2.5. By (4.7) and the definition of $I$,

$$
I(v)>c+\epsilon \quad \text { for } v \in \partial_{M^{\infty}} D_{0},
$$

and we have

$$
D_{2}=\left\{v \in D_{0}: I(v) \leq c+\epsilon\right\} \subset \operatorname{int}_{M^{\infty}} D_{0},
$$

Here we fix an open neighborhood $D_{1}$ of $D_{2}$ in $M^{\infty}$ such that

$$
D_{2} \subset \operatorname{int}_{M \infty} D_{1} \subset \operatorname{cl}\left(D_{1}\right) \subset i n t_{M \infty} D_{0}
$$

and set

$$
W_{i}=\left\{t v: t \in[-\rho+1, \rho+1], v \in D_{i}\right\}, \mathrm{i}=1,2 .
$$

Then

$$
U_{1} \subset W_{2} \subset W_{1} \subset I_{c+\epsilon} \cap U
$$

We note that

$$
I\left(\lambda_{0}(v) v\right)>c+\epsilon \quad \text { for } v \in D_{0} \backslash D_{2} .
$$

Let $V_{1}$ be a Lipschitz continuous vector field associate with $\nabla I$ and $V_{2}$ be a vector field defined on $\left(I_{c+\epsilon} \cap U\right) \backslash W_{2}$ by

$$
V_{2}(u)= \begin{cases}u & \text { if } \lambda_{0}(u)>1 \\ -u & \text { if } \lambda_{0}(u)<1\end{cases}
$$

Since $\lambda_{0}(u) \neq 1$ on $\left(I_{c+\epsilon} \cap U\right) \backslash W_{2}$ by (4.9), we can see that $V_{2}$ is well defined and continuous on $\left(U \cap I_{c_{1}+\bar{\epsilon}}\right) \backslash W_{2}$. We now set

$$
V(u)=\left\|U_{2,-} \cup U_{2,+}-u\right\|\left(\left\|W_{1}^{c}-u\right\| V_{1}(u)+\left\|W_{2}-u\right\| V_{2}(u)\right)
$$

Then $V$ is a Lipschitz continuous vector field on $I_{c+\epsilon} \cap U$ and the solution $\eta$ of (2.14) defines a semiflow. We shall see that

$$
\eta(t, v) \in I_{c+\epsilon} \cap U \quad \text { for all }(t, v) \in[0, \infty) \times\left(I_{c+\epsilon} \cap U\right) .
$$

We first note that from the definition of $V,\langle\nabla I(v), V(v)\rangle>0$ on $I_{c+\epsilon} \cap U$. Then it follows that $\eta(t, v) \leq \eta(s, v)$ for all $t>s \geq 0$ and $v \in I_{c+\epsilon} \cap U$. Since $W_{1} \backslash\left(U_{1,-} \cup U_{1,+}\right) \subset \operatorname{int}\left(I_{c+\epsilon} \cap U\right)$, to show (4.10), it is sufficient to show that (4.10) holds for all $v \in W_{1}^{c} \cap\left(I_{c+\epsilon} \cap U\right)$. If $v \in W_{1}^{c} \cap\left(I_{c+\epsilon} \cap U\right)$, then 
from the definition of $V$, we can see that $\eta(t, v) \in W_{1}^{c} \cap\left(I_{c+\epsilon} \cap U\right)$ for $t \geq 0$ and then (4.10) holds. Moreover we have that for each $v \in W_{1}^{c} \cap\left(I_{c+\epsilon} \cap U\right)$, $\eta(t, v) \in U_{1,-} \cup U_{1,+}$ for $t$ sufficiently large. On the other hand, it follows from the definition of $V$ that

$$
\inf \left\{\|V(u)\|: u \in\left(I_{c+\epsilon} \cap U\right) \backslash \widetilde{U}_{2}\right\}>0 .
$$

Then we can see that for any $v \in I_{c+\epsilon} \cap U$, there exists $t_{v} \geq 0$ such that $\eta(t, v) \in \widetilde{U}_{1}$ for all $t \geq t_{v}$. This completes the proof.

We lastly show that (6) holds. (6) is a consequence of the following Lemma.

Lemma 4.3. If $\left(I_{c+\epsilon} \cap U\right) \backslash K$ is disconnected, then $H_{N-1}\left(\left(I_{c+\epsilon} \cap U\right) \backslash K\right)=$ 2.

Proof. Let $V_{ \pm}$be the components of $\left(I_{c+\epsilon} \cap U\right) \backslash K$ containing $U_{1, \pm}$, respectively. We will see that $\left(I_{c+\epsilon} \cap U\right) \backslash K$ consists of exactly two components $V_{ \pm}$and that $V_{ \pm} \cong S^{N-1}$. Let $v \in D_{0}$. Then from the definition of $M$ and $U$, we have that

$$
\begin{aligned}
&\{t v: t \in[-\rho+1, \rho+1]\} \cap\left(I_{c+\epsilon} \backslash K\right) \\
&=\left\{t v: t \in\left[-\rho+1, t_{1}(v)\right] \cup\left[t_{2}(v), \rho+1\right]\right\},
\end{aligned}
$$

where $-\rho / 2+1 \leq t_{1}(v) \leq t_{2}(v) \leq \rho / 2+1$. This implies that if $t_{1}(v)=t_{2}(v)$ for some $v \in D_{0}$, then $\left(I_{c+\epsilon} \cap U\right) \backslash K$ is connected. Therefore $t_{1}(v)<t_{2}(v)$ for all $v \in D_{0}$. Then, again by (4.12), $\left(I_{c+\epsilon} \cap U\right) \backslash K \cong U_{1,+} \cup U_{1},-$. Then since $U_{1, \pm} \cong S^{N-1}$, the assertion follows.

\section{Appendix}

We put $\mathcal{C}=\cup\left\{\tau_{x} u_{\infty}: x \in R^{N}\right\}$ and

$$
T_{u_{\infty}}(\mathcal{C})=\left\{\lim _{t \rightarrow 0}\left(u_{\infty}(\cdot+t x)-u_{\infty}(\cdot)\right) / t: x \in R^{N}\right\}
$$

It is obvious that $\operatorname{dim} T_{u_{\infty}}(\mathcal{C})=N$. We denote by $\widetilde{H}$ the subspace such that $\widetilde{H} \oplus T_{u_{\infty}}(\mathcal{C})$. Then $H=\tau_{x} \widetilde{H} \oplus \tau_{x} T_{u_{\infty}}(\mathcal{C})$ for each $x \in R^{N}$. For each $r>0$, we set $B_{r}^{0}=B_{r} \cap \widetilde{H}$. Since $\mathcal{C}$ is a smooth N-manifold, we have that there exists a positive number $r_{0}<\left\|u_{\infty}\right\| / 4$ such that for $x, y \in R^{N}$ with $x \neq y$,

$$
\tau_{x}\left(u_{\infty}+B_{r_{0}}^{0}\right) \cap \tau_{y}\left(u_{\infty}+B_{r_{0}}^{0}\right)=\phi
$$

We choose a closed contractible neighborhood $S_{0}$ of $u_{\infty}$ in $M^{\infty} \cap\left(u_{\infty}+\right.$ $B_{r_{0}}^{0}$ ) and $0<\rho<1$ such that

$$
\sup \left\{I^{\infty}(( \pm \rho / 2+1) v): v \in S_{0}\right\}<c
$$


Since $I(v)>c$ for all $v \in S_{0} \backslash\left\{u_{\infty}\right\}$, we have that

$$
\inf \left\{I^{\infty}(v): v \in \partial_{M \infty \cap\left(u_{\infty}+B_{r_{0}}^{0}\right)} S_{0}\right\}>c .
$$

Here we recall that mappings $t \rightarrow I^{\infty}(( \pm t+1) v)$ are decreasing as $t$ varies from 0 to $\pm \rho$. Then from (5.2), we have

$$
\begin{aligned}
I_{c}^{\infty} \cap\{t v: & t \in[-\rho+1, \rho+1]\} \\
& =\left\{t v: t \in\left[-\rho+1, \lambda_{-}(v)\right]\right\} \cup\left\{t v: t \in\left[\lambda_{+}(v), \rho+1\right]\right\}
\end{aligned}
$$

where

$$
\begin{cases}\lambda_{-}(v)<1<\lambda_{+}(v) & \text { for } v \in S_{0} \backslash\left\{u_{\infty}\right\} \\ \lambda_{-}(v)=\lambda_{+}(v)=1 & \text { for } v=u_{\infty} .\end{cases}
$$

That is, for each $v \in S_{0} \backslash\left\{u_{\infty}\right\}$, the set $I_{c}^{\infty} \cap\{t v: t \in[-\rho+1, \rho+1]\}$ consists of two intervals, and each interval has one end point in one of the sets

$$
V_{ \pm}=\left\{( \pm \rho+1) v: v \in S_{0}\right\} .
$$

Then noting that $\lambda_{-}(\cdot)$ and $\lambda_{+}(\cdot)$ are continuous and $V_{ \pm}$are contractible, we have from observations above that

$$
I_{c}^{\infty} \cap\left(V \backslash\left\{u_{\infty}\right\}\right) \cong V_{-} \cup V_{+} \cong\{0,1\} \text { and } I_{c}^{\infty} \cap V \cong[0,1]
$$

Now let $0<\epsilon<c$. First we note that

$$
I^{\infty}(u)=\tau_{x} \cdot I^{\infty}(u)=I^{\infty}\left(\tau_{x} u\right) \quad \text { for all } x \in R^{N} \text { and } u \in H .
$$

Then we have that $I_{c}^{\infty} \cap\left(\cup\left\{\tau_{x} V: x \in R^{N}\right\}\right)$ and $I_{\epsilon}^{\infty} \cap\left(\cup\left\{\tau_{x} V: x \in R^{N}\right\}\right)$ have the same homotopy type with that of $I_{c}^{\infty} \cap V$ and $I_{\epsilon}^{\infty} \cap V$, respectively. On the other hand, by the same argument for the second deformation lemma in Chang [4], we have that $I_{c}^{\infty}$ is a strong deformation rectraction of $I_{c+\epsilon}^{\infty}$. Then we find

$$
H_{q}\left(I_{c+\epsilon}^{\infty}, I_{c-\epsilon}^{\infty}\right) \cong H_{q}\left(I_{c}^{\infty}, I_{c-\epsilon}^{\infty}\right) .
$$

We also have by the deformation property that

$$
H_{q}\left(I_{c}^{\infty} \backslash \mathcal{C}, I_{c-\epsilon}^{\infty}\right) \cong H_{q}\left(I_{c-\epsilon}^{\infty}, I_{c-\epsilon}^{\infty}\right) \cong 0
$$

From the exactness of the singular homology groups, we have

$$
\begin{aligned}
H_{q}\left(I_{c}^{\infty} \backslash \mathcal{C}, I_{c-\epsilon}\right) & \rightarrow H_{q}\left(I_{c}^{\infty}, I_{c-\epsilon}^{\infty}\right) \\
& \rightarrow H_{q}\left(I_{c}^{\infty}, I_{c}^{\infty} \backslash \mathcal{C}\right) \rightarrow H_{q-1}\left(I_{c}^{\infty} \backslash \mathcal{C}, I_{c-\epsilon}^{\infty}\right) \rightarrow \cdots
\end{aligned}
$$

and we find

$$
0 \rightarrow H_{q}\left(I_{c}^{\infty}, I_{c-\epsilon}^{\infty}\right) \rightarrow H_{q}\left(I_{c}^{\infty}, I_{c}^{\infty} \backslash \mathcal{C}\right) \rightarrow 0
$$


That is,

$$
H_{q}\left(I_{c}^{\infty}, I_{c-\epsilon}^{\infty}\right) \cong H_{q}\left(I_{c}^{\infty}, I_{c}^{\infty} \backslash \mathcal{C}\right) .
$$

Then from the excision property of homology groups and (5.5), we have

$$
\begin{aligned}
H_{*}\left(I_{c+\epsilon}^{\infty}, I_{\epsilon}^{\infty}\right) & \cong H_{*}\left(I_{c}^{\infty}, I_{c}^{\infty} \backslash \mathcal{C}\right) \\
& \cong H_{*}\left(I_{c}^{\infty} \cap\left(\cup_{x} \tau_{x} V\right), I_{c}^{\infty} \cap\left(\left(\cup_{x} \tau_{x} V\right) \backslash \mathcal{C}\right)\right) \\
& \cong H_{*}\left(I_{c}^{\infty} \cap V, I_{c}^{\infty} \cap\left(V \backslash\left\{u_{\infty}\right\}\right)\right) \\
& \cong H_{*}([0,1],\{0,1\})
\end{aligned}
$$

This completes the proof.

\section{REFERENCES}

1. V. Benci and G. Cerami, Positive solutions of some nonlinear elliptic problems in exterior domains, Arch. Rational Mech. Anal. 99 (1987), 283-300..

2. H. Berestycki and P. L. Lions, Nonlinear scalar field equations, I, II, Arch. Rational Mech. Anal. 82 (1982), 313-376.

3. D. M. Cao, Positive solutions and bifurcation from the essential spectrum of a semilinear elliptic equation on $R^{N}$, Nonlinear Anal. TMA 15 (1990), 1045-1052.

4. K. C. Chang, Indefinite dimensional Morse theory and its applications, Séminaire de Mathématiques Supérieures, 97, Univ. de Montreal (1985).

5. W. Y. Ding and W. M. Ni, On the existence of positive solutions of a semilinear elliptic equation, Arch. Rational Mech. Anal. 91 (1986), 283-307.

6. N. Hirano, Existence of entire positive solutions for nonhomogenuous elliptic equations (to appear).

7. M. K. Kwong, Uniquness of positive solutions of $\Delta u-u+u^{p}=0$ in $R^{N}$, Arch. Rational Mech. Anal. 105 (1989), 243-266.

8. P. L. Lions, The concentration-compactness principle in the calculus of variations, the locally compact case. Part I, Ann. Inst. H. Poincaré Anal. Non Linéaire 1 (1984), 109-145.

9. P. L. Lions, The concentration-compactness principle in the calculus of variations, the locally compact case. Part II, Ann. Inst. H. Poincaré Anal. Non Linéaire 1 (1984), 223-283.

10. H. P. Rabinowitz, Variational methods for nonlinear eigenvalue problems, Proc. Sympos. on Eigenvalues of Nonlinear Problems (1974), Edizioni Cremonese, Rome, 141195.

11. E. Spanier, Algebraic Topology, McGraw-Hill, New York, 1966.

12. W. A. Strauss, Existence of solitary waves in higher dimensions, Comm. Math. Phys. 55 (1977), 149-162.

13. C. A. Stuart, Bifurcation from the essential spectrum for some noncompact nonlinearities (to appear).

Department of Mathematics, Faculty of Engineering, Yokohama National University, Tokiwadai, Hodogayaku, Yokohama Japan

E-mail address: hirano@sci.math.ynu.ac.jp 


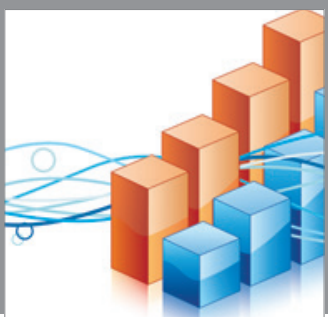

Advances in

Operations Research

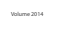

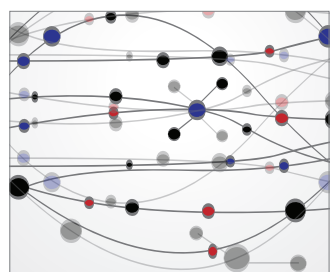

\section{The Scientific} World Journal
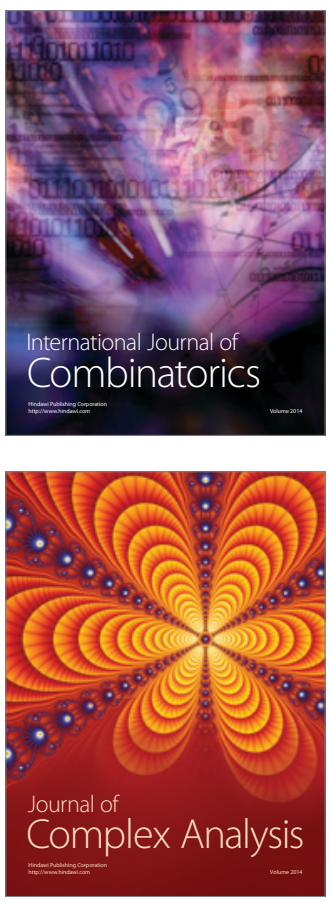

International Journal of

Mathematics and

Mathematical

Sciences
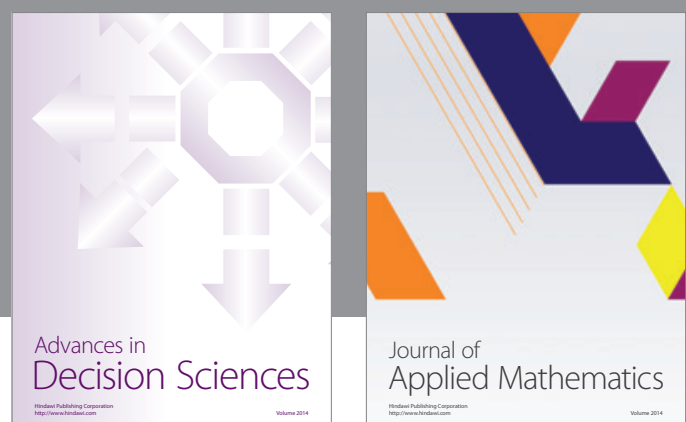

Journal of

Applied Mathematics
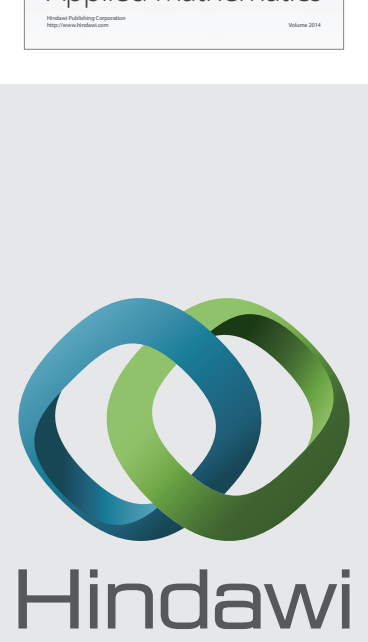

Submit your manuscripts at http://www.hindawi.com
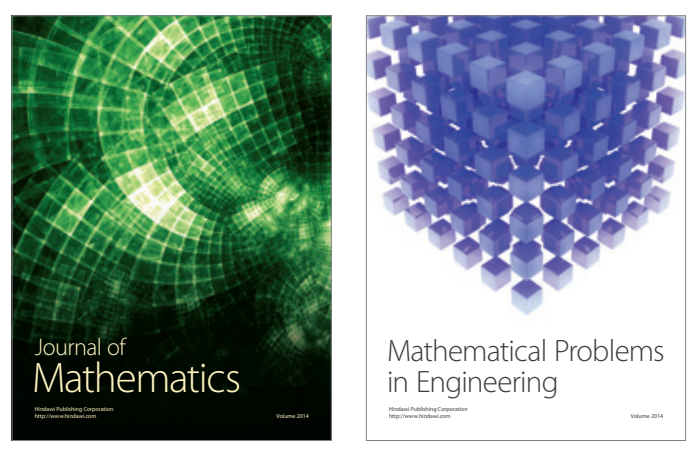

Mathematical Problems in Engineering
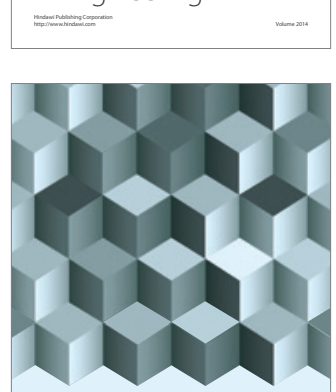

Journal of

Function Spaces
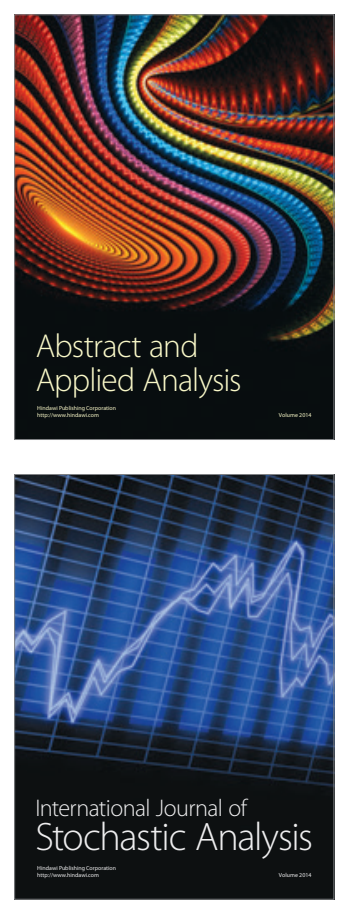

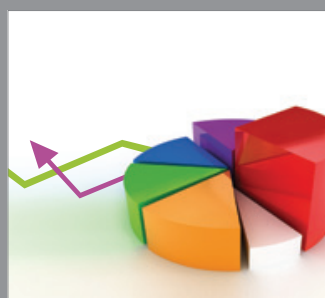

ournal of

Probability and Statistics

Promensencen
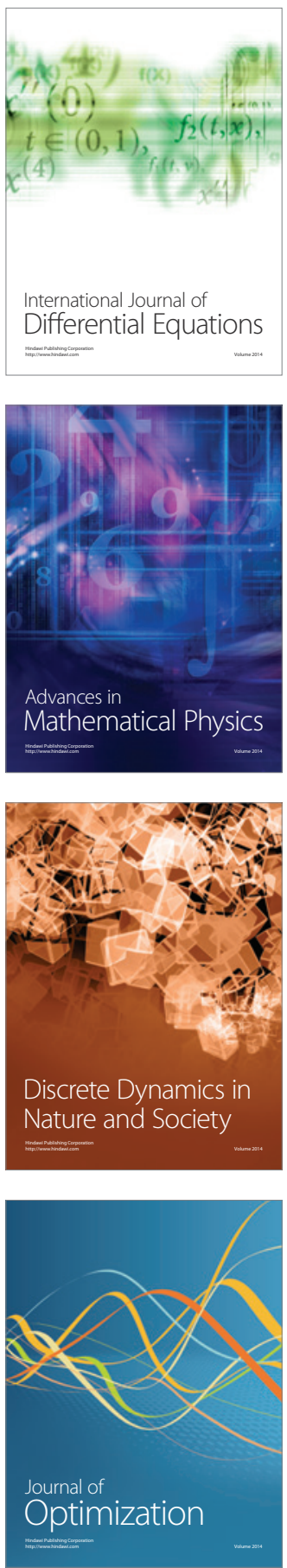